\title{
Integrability of Nonholonomic Heisenberg Type Systems
}

Yury A. GRIGORYEV ${ }^{\dagger}$, Alexey P. SOZONOV ${ }^{\dagger}$ and Andrey V. TSIGANOV ${ }^{\dagger \dagger}$

$\dagger$ St. Petersburg State University, St. Petersburg, Russia

E-mail:yury.grigoryev@gmail.com,sozonov.alexey@yandex.ru,andrey.tsiganov@gmail.com

$\ddagger$ Udmurt State University, Izhevsk, Russia

Received March 17, 2016, in final form November 22, 2016; Published online November 25, 2016

http://dx.doi.org/10.3842/SIGMA.2016.112

\begin{abstract}
We show that some modern geometric methods of Hamiltonian dynamics can be directly applied to the nonholonomic Heisenberg type systems. As an example we present characteristic Killing tensors, compatible Poisson brackets, Lax matrices and classical $r$ matrices for the conformally Hamiltonian vector fields obtained in a process of reduction of Hamiltonian vector fields by a nonholonomic constraint associated with the Heisenberg system.
\end{abstract}

Key words: Hamiltonian dynamics; nonholonomic systems

2010 Mathematics Subject Classification: 37J60; 70G45; 70H45

\section{Introduction}

Hamiltonian mechanics emerged in 1833 as a convenient reformulation of classical Newtonian mechanics and analytical Lagrangian mechanics. Over the next almost two centuries, it was gradually realized that Hamiltonian formulation of a system of differential equations

$$
\frac{d}{d t} x_{k}=X_{k}, \quad X=P d H
$$

has several advantages based on the main postulate that evolution of a physical system over time is governed by a single Hamiltonian $H$ of that system and a Poisson bivector $P$ describing geometry and topology of the phase space. Many different mathematical methods and concepts are widely used in the Hamiltonian formalism. For instance, we can construct first integrals of the Hamiltonian vector field $X$ using Killing tensors (Riemannian geometry), compatible Poisson brackets (bi-Hamiltonian geometry) or Lax matrices (algebraic geometry, Lie algebras theory, classical $r$-matrix theory etc.).

It is natural to ask what happens to these mathematical methods when we impose nonholonomic constraints on the Hamiltonian system and make a suitable reduction. Usually presence of the constraints drastically modifies or destroys these geometric constructions even when one gets a conformally Hamiltonian vector field

$$
\hat{X}=\mu(x) \hat{P} d \hat{H}
$$

after the reduction, see $[2,5,7,8,9,10,11,15,22,24]$ and references within. Here $\hat{H}$ and $\hat{P}$ are Hamiltonian and Poisson bivector on reduced phase space, whereas function $\mu(x)$ is the so-called conformal factor.

The main aim of this note is to present a family of conformally Hamiltonian dynamical systems for which all the geometric methods listed above can be applied without any additional modifications. The corresponding nonholonomic constraint is associated with the socalled Heisenberg system or nonholonomic integrator, which plays an important role in both 
nonlinear control and nonholonomic dynamics [7]. We have to underline that the integrable systems discussed in this note look quite artificial from the viewpoint of control theory and mechanics but they provide an example of the standard geometric methods applicability to the conformally Hamiltonian systems.

This paper is organized as follows. Section 1.1 recalls a brief description of the constrained motion in three-dimensional Euclidean space. Section 2 contains the main results on reduced motion of the nonholonomic Heisenberg type systems on the plane. We will show that integrable potentials for this non Hamiltonian vector field satisfy to the Bertrand-Darboux type equation. Solutions of this equation and the corresponding characteristic coordinates will be explicitly determined. Section 3 deals with application of the standard Stäckel theory to the conformally Hamiltonian vector field. We will discuss a construction of the Stäckel matrices, compatible Poisson structures, Lax matrices and classical $r$-matrices.

\subsection{Main definitions}

According to $[7,18,24]$ we take the standard Hamiltonian equations of motion in Euclidean space $\mathbb{R}^{3}$

$$
\dot{q}_{i}=\frac{\partial H}{\partial p_{i}}, \quad \dot{p}_{i}=-\frac{\partial H}{\partial q_{i}}, \quad i=1,2,3,
$$

where

$$
H=\frac{1}{2}\left(p_{1}^{2}+p_{2}^{2}+p_{3}^{2}\right)+V\left(q_{1}, q_{2}, q_{3}\right) .
$$

On the phase space $M=T^{*} \mathbb{R}^{3}$ we can introduce coordinates $x=(q, p)$ in which equations (1.1) have form $\dot{x}_{i}=X_{i}$ and to determine Hamiltonian vector field

$$
X=\sum_{i=1}^{6} X_{i} \frac{\partial}{\partial x_{i}},
$$

which is a linear operator on a space of the smooth functions on $M$ that encodes the evolution of any quantity

$$
\dot{F}=X(F)=\sum_{i=1}^{6} X_{i} \frac{\partial F}{\partial x_{i}} .
$$

Let us impose the constraint of first order in momenta (velocities)

$$
f=(b, p)=0,
$$

where $b=\left(b_{1}, b_{2}, b_{3}\right)$ is a vector depending on coordinates $q$ and $(x, y)$ means an inner product in $\mathbb{R}^{3}$. In this case equations of motion are written in the following form

$$
\dot{q}_{i}=\frac{\partial H}{\partial p_{i}}, \quad \dot{p}_{i}=-\frac{\partial H}{\partial q_{i}}+\lambda b_{i}, \quad i=1,2,3,
$$

together with the constraint equation (1.3). The corresponding vector field looks like an additive perturbation of the initial Hamiltonian vector field

$$
\hat{X}=\left.X\right|_{b=0}+\lambda\left(b_{1} \frac{\partial}{\partial p_{1}}+b_{2} \frac{\partial}{\partial p_{2}}+b_{3} \frac{\partial}{\partial p_{3}}\right),
$$


where unknown Lagrange multiplier $\lambda$ has to be computed from the condition

$$
\dot{f}=\hat{X}(f)=\left.X(f)\right|_{b=0}+\lambda(b, b)=0,
$$

such that

$$
\lambda=\frac{\left.X(f)\right|_{b=0}}{(b, b)} .
$$

Here $\left.X(f)\right|_{b=0}$ denotes the vector field in the absence of constraint. Such equations and an equivalence of Hamiltonian and Lagrangian reductions are carefully discussed in the book [7].

For completeness and self-sufficiency of presentation we consider integrable constraint

$$
f=p_{3}=0, \quad b=(0,0,1),
$$

when a third component of momenta is equal to zero, and non integrable constraint

$$
f=p_{3}-\left(q_{2} p_{1}-q_{1} p_{2}\right)=0, \quad b=\left(-q_{2}, q_{1}, 1\right)
$$

when third components of momenta and angular momenta coincide with each other.

If we assume that the potential $V$ in $\mathbb{R}^{3}$ does not depend on $q_{3}$, then Lagrange multipliers (1.5) are equal to

$$
\lambda=0 \quad \text { and } \quad \lambda=\frac{q_{1} \partial_{2} V-q_{2} \partial_{1} V}{1+q_{1}^{2}+q_{2}^{2}}, \quad \partial_{j}=\frac{\partial}{\partial q_{j}},
$$

respectively. Thus, the Hamiltonian system (1.2) with integrable constraint (1.6) represent a Hamiltonian system on the plane $\mathbb{R}^{2}$ embedded in a three dimensional Euclidean space $\mathbb{R}^{3}$ in which there is no force acting on the third component. If we impose non integrable constraint (1.7), the equations for $q_{3}$ also decouples from the rest of the system (1.4) and we obtain a two-degrees of freedom non-Hamiltonian system on the plane. Following to $[7,18]$ we will call dynamical system associated with $\hat{X}$ as a Heisenberg type system.

Integrability of the Hamiltonian systems on the plane is the well-known problem of classical mechanics considered by Bertrand [4] and Darboux [12]. Later on results of this investigation due to Darboux were included in the textbook of Whittaker on analytical mechanics [25] almost verbatim. In the next Section we compare classical Bertrand-Darboux theorem with its nonHamiltonian counterpart, which appears in the nonholonomic case.

\section{Reduced systems on the plane}

When $V=V\left(q_{1}, q_{2}\right)$, substituting integrable constraint $p_{3}=0$ (1.6) into (1.2) one gets

$$
H_{1}=\frac{1}{2}\left(p_{1}^{2}+p_{2}^{2}\right)+V\left(q_{1}, q_{2}\right)=\frac{1}{2} \mathrm{~g}^{i j} p_{i} p_{j}+V\left(q_{1}, q_{2}\right), \quad \mathrm{g}=\left(\begin{array}{ll}
1 & 0 \\
0 & 1
\end{array}\right)
$$

with the summation convention in force; we regard the $\mathrm{g}^{i j}$ as components of the covariant form of the metric tensor $g$ on the plane, and use this metric freely to raise and lower indices.

After standard Hamiltonian reduction by cyclic third coordinate, the original Hamiltonian vector field (1.1) in $T^{*} \mathbb{R}^{3}$ becomes the Hamiltonian vector field in $T^{*} \mathbb{R}^{2}$

$$
\dot{x}=X, \quad X=P d H_{1}, \quad P=\left(\begin{array}{cc}
0 & I \\
-I & 0
\end{array}\right),
$$


where $x=\left(q_{1}, q_{2}, p_{1}, p_{2}\right)$. The Poisson bivector $P$ in (2.2) defines canonical Poisson bracket

$$
\left\{q_{i}, p_{i}\right\}=1, \quad\left\{q_{i}, p_{k}\right\}=\left\{p_{1}, p_{2}\right\}=0, \quad i \neq k .
$$

Of course, this vector field $X$ preserves Poisson structure $P$, energy $H_{1}$ and the standard volume 2 -form $\Omega=d q \wedge d p$.

Substituting non integrable constraint $p_{3}=q_{2} p_{1}-q_{1} p_{2}$ (1.7) into (1.2) one gets

$$
\begin{aligned}
& \hat{H}_{1}=\frac{1}{2}\left(p_{1}^{2}+p_{2}^{2}+\left(q_{2} p_{1}-q_{1} p_{2}\right)^{2}\right)+V\left(q_{1}, q_{2}\right)=\frac{1}{2} \hat{\mathrm{g}}^{i j} p_{i} p_{j}+V\left(q_{1}, q_{2}\right), \\
& \hat{\mathrm{g}}=\left(\begin{array}{cc}
1+q_{2}^{2} & -q_{1} q_{2} \\
-q_{1} q_{2} & 1+q_{1}^{2}
\end{array}\right) .
\end{aligned}
$$

The second order contravariant symmetric tensor field $\hat{g}$ is a Killing tensor with respect to the standard metric tensor g, i.e., it satisfies to the Killing equation

$$
\llbracket \mathrm{g}, \hat{\mathrm{g}} \rrbracket=0
$$

where $\llbracket \cdot, \cdot \rrbracket$ is a Schouten bracket. It allows us to construct conformal Killing tensor of second order

$$
G=\hat{\mathrm{g}}-\operatorname{tr}(\hat{\mathrm{g}}) \mathrm{g}=-\left(\begin{array}{cc}
1+q_{1}^{2} & q_{1} q_{2} \\
q_{1} q_{2} & 1+q_{2}^{2}
\end{array}\right)
$$

with vanishing Nijenhuis torsion and to define the standard Turiel deformation of the standard symplectic form

$$
\hat{\omega}=d\left(G^{i j} p_{i} d q_{j}\right),
$$

see $[21,22]$ and references within. The corresponding Poisson bracket

$$
\left\{q_{i}, p_{i}\right\}^{\wedge}=1+q_{i}^{2}, \quad\left\{q_{i}, p_{j}\right\}^{\wedge}=q_{1} q_{2}, \quad\left\{p_{1}, p_{2}\right\}^{\wedge}=q_{1} p_{2}-q_{2} p_{1}, \quad i \neq j
$$

is compatible with canonical bracket (2.3). Because the Turiel deformation is trivial deformation in the Lichnerowicz-Poisson sense, there is a change of variables

$$
p_{1} \rightarrow \pi_{1}=\frac{q_{1} q_{2} p_{2}-\left(1+q_{2}^{2}\right) p_{1}}{1+x_{1}^{2}+x_{2}^{2}}, \quad p_{2} \rightarrow \pi_{2}=\frac{q_{1} q_{2} p_{1}-\left(1+q_{1}^{2}\right) p_{2}}{1+x_{1}^{2}+x_{2}^{2}},
$$

which transforms this bracket to the canonical one (2.3), similar to other nonholonomic systems with constraints of first order in momenta $[6,10,20]$.

After the nonholonomic reduction, original Hamiltonian vector field (1.1) in $T^{*} \mathbb{R}^{3}$ becomes a conformally Hamiltonian vector field in $T^{*} \mathbb{R}^{2}$

$$
\dot{x}=\hat{X}, \quad \hat{X}=\mu \hat{P} d \hat{H}_{1}, \quad \hat{P}=\hat{\omega}^{-1},
$$

where conformal factor

$$
\mu=\left(1+q_{1}^{2}+q_{2}^{2}\right)^{-1}
$$

is a nowhere vanishing smooth function on the plane. This vector field possesses energy $\hat{H}_{1}$ and the volume 2 -form $\hat{\Omega}=\mu d q \wedge d p$, but it does not preserve the Poisson bivector $\hat{P}$. 


\subsection{Bertrand-Darboux type equation}

An existence of the integrals of second order in velocities for Hamiltonian vector field (2.2) is described by a classical Bertrand-Darboux theorem [4, 12, 25].

Proposition 1. The function (2.1)

$$
H_{1}=\frac{1}{2} \mathrm{~g}^{i j} p_{i} p_{j}+V\left(q_{1}, q_{2}\right), \quad \mathrm{g}=\left(\begin{array}{ll}
1 & 0 \\
0 & 1
\end{array}\right)
$$

defines integrable Hamiltonian vector field $X$ (2.2), which has an independent quadratic first integral

$$
H_{2}=K^{i j} p_{i} p_{j}+U\left(q_{1}, q_{2}\right),
$$

if the second order contravariant symmetric tensor field $K$ obeys the Killing tensor equation

$$
\llbracket \mathrm{g}, K \rrbracket=0,
$$

and potential $V$ satisfies the compatibility condition

$$
d(\mathbf{K} d V)=0, \quad \mathbf{K}=K \mathrm{~g}^{-1},
$$

where $\mathbf{K}$ is the tensor field of $(1,1)$ type.

A characteristic coordinate system for (2.11) provides separation for the potential $V$ and can be taken as one of the following four orthogonal coordinate systems on the plane: elliptic, parabolic, polar or Cartesian.

If a non-trivial solution exists, the Bertrand-Darboux equation (2.11) can be reduced to canonical form by transforming to characteristic coordinates, which appear to be separation coordinates for the Hamilton-Jacobi equation related to the natural Hamiltonian (see Bertrand [4], Darboux [12], or Whittaker [25] and Ankiewicz and Pask [1] for a full proof of the BD theorem). The modern proof of this classic statement may be found in [19].

In nonholonomic case we can also substitute the same ansatz (2.9) into the equation $\dot{H}_{2}=0$ and get the following generalisation of the Bertrand-Darboux result.

Proposition 2. The function (2.4)

$$
\hat{H}_{1}=\frac{1}{2} \hat{\mathrm{g}}^{i j} p_{i} p_{j}+V\left(q_{1}, q_{2}\right), \quad \hat{\mathrm{g}}=\left(\begin{array}{cc}
1+q_{2}^{2} & -q_{1} q_{2} \\
-q_{1} q_{2} & 1+q_{1}^{2}
\end{array}\right)
$$

defines integrable conformally Hamiltonian vector field $\hat{X}(2.7)$, which has an independent quadratic first integral

$$
\hat{H}_{2}=K^{i j} p_{i} p_{j}+U\left(q_{1}, q_{2}\right),
$$

if the second order contravariant symmetric tensor field $K$ is a Killing tensor

$$
\llbracket \mathrm{g}, K \rrbracket=0, \quad \mathrm{~g}=\left(\begin{array}{ll}
1 & 0 \\
0 & 1
\end{array}\right)
$$

and potential $V$ satisfies the compatibility condition

$$
d(\hat{\mathbf{K}} d V)=0, \quad \hat{\mathbf{K}}=K \hat{\mathrm{g}}^{-1} .
$$

A characteristic coordinate system for (2.15) provides separation for potential $V\left(q_{1}, q_{2}\right)$. 
In this case existence of the second integral of motion guarantees integrability of the given non Hamiltonian vector field $\hat{X}$ according to the Euler-Jacobi theorem [17]. Equation (2.15) was briefly discussed in [23] using coordinates $q_{1,2}$ and momenta $\pi_{1,2}(2.6)$.

Remark 1. In contrast with the standard Bertrand-Darboux theorem the Killing equation (2.14) and compatibility condition (2.15) include two different metrics $g$ and $\hat{g}$. It happens because the reaction force is entered in the second part of the vector field (1.4) only. Sequentially, $K$ is a Killing tensor with respect to the Euclidean metric $g$ to the plane, which is an induced metric associated with standard embedding $\mathbb{R}^{2} \subset \mathbb{R}^{3}$ in Cartesian coordinates. However, $K$ is not a Killing tensor with respect to metric $\hat{g}$, which appears in the reduced Hamiltonian (2.4) after nonholonomic reduction and then defines raising and lowering indices in the compatibility condition (2.15).

\subsection{Solutions of the Bertrand-Darboux type equation}

In [12] Darboux proceeds to find the unknown potential $V\left(q_{1}, q_{2}\right)$ by solving the compatibility condition (2.11) using method of characteristics. Solving the second compatibility condition (2.15) we can apply the same method.

In both cases characteristic coordinates for (2.11) and (2.15) consist of eigenvalues of the corresponding $(1,1)$ tensor field $\mathbf{K}$ or $\hat{\mathbf{K}}$. In order to describe these coordinates we can start with a well-known generic solution of the common Killing tensor equations (2.10) and (2.14)

$$
K=\left(\begin{array}{cc}
c_{1} q_{2}^{2}+2 c_{2} q_{2}+c_{3} & -c_{1} q_{1} q_{2}-c_{2} q_{1}-c_{4} q_{2}+c_{6} \\
-c_{1} q_{1} q_{2}-c_{2} q_{1}-c_{4} q_{2}+c_{6} & c_{1} q_{1}^{2}+2 c_{4} q_{1}+c_{5}
\end{array}\right),
$$

which depends on six constants of integration $c_{1}, \ldots, c_{6}$.

Substituting this generic solution into the standard compatibility condition (2.11) one gets

$$
\left(A \partial_{11}+B \partial_{22}+C \partial_{12}+a \partial_{1}+b \partial_{2}\right) V\left(q_{1}, q_{2}\right)=0
$$

where $\partial_{i}=\partial / \partial q_{i}, \partial_{i k}=\partial^{2} / \partial q_{i} \partial q_{k}$ and polynomials of second order read as

$$
A=c_{1} q_{1} q_{2}+c_{2} q_{1}+c_{4} q_{2}-c_{6}, \quad B=-A, \quad C=c_{1} q_{2}^{2}-c_{1} q_{1}^{2}+2 c_{2} q_{2}-2 c_{4} q_{1}+c_{3}-c_{5},
$$

whereas polynomials of the first order are

$$
a=3\left(c_{1} q_{2}+c_{2}\right), \quad b=-3\left(c_{1} q_{1}+c_{4}\right) .
$$

The linear PDE (2.17) of second order was obtained by Bertrand [4] and studied by Darboux [12]. Thus, it was later called the Bertrand-Darboux equation [19, 25].

Solving (2.17) for $V\left(q_{1}, q_{2}\right)$ amounts to finding admissible potentials of the Hamiltonian systems defined by $H$ (2.1), which integrability is afforded by the existence of first integrals (2.9) which are quadratic in the momenta. Second potential $U\left(q_{1}, q_{2}\right)$ in $(2.9)$ is a solution of the equation

$$
d(\mathbf{K} d U)=V .
$$

In nonholonomic case, substituting the same generic solution (2.16) into the compatibility condition (2.15) one gets similar linear PDE

$$
\left(1+q_{1}^{2}+q_{2}^{2}\right)\left(\hat{A} \partial_{11}+\hat{B} \partial_{22}+\hat{C} \partial_{12}\right) V\left(q_{1}, q_{2}\right)+\left(\hat{a} \partial_{1}+\hat{b} \partial_{2}\right) V\left(q_{1}, q_{2}\right)=0,
$$

with coefficients

$$
\hat{A}=\left(1+q_{1}^{2}\right)\left(q_{1} c_{2}-c_{6}\right)+q_{2}\left(1-q_{1}^{2}\right) c_{4}+q_{1} q_{2}\left(c_{1}-c_{5}\right),
$$




$$
\begin{aligned}
& \hat{B}=-\left(1+q_{2}^{2}\right)\left(q_{2} c_{4}-c_{6}\right)-q_{1}\left(1-q_{2}^{2}\right) c_{2}-q_{1} q_{2}\left(c_{1}-c_{3}\right), \\
& \hat{C}=-\left(q_{1}^{2}-q_{2}^{2}\right) c_{1}+\left(1+q_{1}^{2}\right)\left(2 q_{2} c_{2}+c_{3}\right)-\left(1+q_{2}^{2}\right)\left(2 q_{1} c_{4}+c_{5}\right),
\end{aligned}
$$

and

$$
\begin{aligned}
\hat{a}= & \left(q_{1}^{2}+q_{2}^{2}+3\right)\left(c_{1} q_{2}+2 c_{2} q_{1}^{2}-2 c_{4} q_{1} q_{2}\right)-q_{2}\left(2\left(q_{1}^{2}+1\right) c_{3}-\left(q_{1}^{2}-q_{2}^{2}-1\right) c_{5}\right) \\
& +q_{1}\left(q_{1}^{2}-3 q_{2}^{2}+1\right) c_{6}-\left(q_{1}^{2}+q_{2}^{2}-3\right) c_{2}, \\
\hat{b}= & -\left(q_{1}^{2}+q_{2}^{2}+3\right)\left(c_{1} q_{1}+2 c_{4} q_{2}^{2}-2 c_{2} q_{1} q_{2}\right)+q_{1}\left(2\left(q_{2}^{2}+1\right) c_{5}+\left(q_{1}^{2}-q_{2}^{2}+1\right) c_{3}\right) \\
& +q_{2}\left(3 q_{1}^{2}-q_{2}^{2}-1\right) c_{6}+\left(q_{1}^{2}+q_{2}^{2}-3\right) c_{4} .
\end{aligned}
$$

Solutions $V\left(q_{1} q_{2}\right)$ of this Bertrand-Darboux type equation (2.18) determine all the admissible Jacobi integrals (2.4), which define integrable conformally Hamiltonian vector fields (2.7) with first integrals (2.13) of second order in momenta. In this case potential $U\left(q_{1}, q_{2}\right)$ in (2.13) is a solution of the equation

$$
d(\hat{\mathbf{K}} d U)=V .
$$

Remark 2. Before solving the PDE (2.17), Darboux ingeniously observes that it can be simplified without loss of generality [12]. Indeed, by rotating and translating the axes, one can simplify the general solution of the Killing tensor equation, thus bringing it to a certain canonical form. In modern language solving the equivalence and canonical forms problem for the Killing tensor equations (2.10), (2.14) is equivalent to analysing the orbits of the six-dimensional vector space of solutions under the action of the Lie group of orientation-preserving isometries.

It is well known that there are four types of orbits generated by the following canonical Killing tensors:

$$
\begin{aligned}
& \text { Cartesian: } \quad K^{(1)}=\left(\begin{array}{ll}
1 & 0 \\
0 & 0
\end{array}\right) \text {, } \\
& \text { polar: } \quad K^{(2)}=\left(\begin{array}{cc}
q_{2}^{2} & -q_{1} q_{2} \\
-q_{1} q_{2} & q_{1}^{2}
\end{array}\right) \text {, } \\
& \text { parabolic: } \quad K^{(3)}=\left(\begin{array}{cc}
0 & -q_{2} \\
-q_{2} & 2 q_{1}
\end{array}\right) \text {, } \\
& \text { elliptic: } \quad K^{(4)}=\left(\begin{array}{cc}
\kappa^{2}+q_{2}^{2} & -q_{1} q_{2} \\
-q_{1} q_{2} & q_{1}^{2}
\end{array}\right), \quad \kappa \in \mathbb{R},
\end{aligned}
$$

associated with Cartesian, polar, parabolic and elliptic coordinate systems on the plane, respectively.

Let us consider these characteristic coordinate systems associated with the Killing tensors (2.19).

Elliptic coordinates. Substituting Killing tensor $K^{(4)}$ into the $(1,1)$ tensor $\mathbf{K}=K^{(4)} \mathrm{g}^{-1}$ and calculating its eigenvalues one gets elliptic coordinates

$$
\xi_{1,2}=\kappa+q_{1}^{2}+q_{2}^{2} \pm \sqrt{\kappa^{2}-2\left(q_{1}^{2}-q_{2}^{2}\right) \kappa+\left(q_{1}^{2}+q_{2}^{2}\right)^{2}}
$$

up to the constant factor. In nonholonomic case, substituting the same tensor $K^{(4)}$ into the characteristic tensor $\hat{\mathbf{K}}=K^{(4)} \hat{\mathrm{g}}^{-1}$, one gets the following eigenvalues

$$
\hat{\xi}_{1,2}=\frac{\left(1+q_{1}^{2}\right) \kappa+q_{1}^{2}+q_{2}^{2} \pm \sqrt{\left(1+q_{1}^{2}\right)^{2} \kappa^{2}-2\left(q_{1}^{2}\left(q_{1}^{2}+q_{2}^{2}\right)+q_{1}^{2}-q_{2}^{2}\right) \kappa+\left(q_{1}^{2}+q_{2}^{2}\right)^{2}}}{1+q_{1}^{2}+q_{2}^{2}} .
$$


Solutions of the compatibility equations (2.11) and (2.15) are labelled by two arbitrary functions $F_{1}$ and $F_{2}$ on these coordinates:

$$
V\left(q_{1}, q_{2}\right)=\frac{F_{1}\left(u_{1}\right)-F_{2}\left(u_{2}\right)}{u_{1}-u_{2}}, \quad u_{1,2}=\xi_{1,2} \quad \text { or } \quad u_{1,2}=\hat{\xi}_{1,2} .
$$

Parabolic coordinates. In Hamiltonian case eigenvalues of the characteristic tensor $\mathbf{K}=$ $K^{(3)} \mathrm{g}^{-1}$ are standard parabolic coordinates

$$
\zeta_{1,2}=q_{1} \pm \sqrt{q_{1}^{2}+q_{2}^{2}}
$$

whereas in nonholonomic case eigenvalues of the characteristic tensor $\hat{\mathbf{K}}=K^{(3)} \hat{\mathrm{g}}^{-1}$ read as

$$
\hat{\zeta}_{1,2}=\frac{q_{1} \pm \sqrt{q_{2}^{2}+1} \sqrt{q_{1}^{2}+q_{2}^{2}}}{1+q_{1}^{2}+q_{2}^{2}} .
$$

It is easy to express new characteristic coordinates via standard ones

$$
\hat{\zeta}_{1,2}=\frac{2\left(\zeta_{1}+\zeta_{2} \pm\left(\zeta_{1}-\zeta_{2}\right) \sqrt{1-\zeta_{1} \zeta_{2}}\right)}{\left(\zeta_{1}-\zeta_{2}\right)^{2}+4} .
$$

In both cases the desired separable potentials are equal to

$$
V\left(q_{1}, q_{2}\right)=\frac{F_{1}\left(u_{1}\right)-F_{2}\left(u_{2}\right)}{u_{1}-u_{2}}, \quad u_{1,2}=\zeta_{1,2} \quad \text { or } \quad u_{1,2}=\hat{\zeta}_{1,2}
$$

Polar coordinates. Using one nontrivial eigenvalue of characteristic tensors $\mathbf{K}=K^{(2)} \mathrm{g}^{-1}$ and $\hat{\mathbf{K}}=K^{(2)} \hat{\mathrm{g}}^{-1}$ we can introduce only one coordinate

$$
r=\sqrt{q_{1}^{2}+q_{2}^{2}}, \quad \text { or } \quad \hat{r}=\sqrt{\frac{q_{1}^{2}+q_{2}^{2}}{1+q_{1}^{2}+q_{2}^{2}}} .
$$

The second coordinate is a function on $q_{1} / q_{2}$, for instance, it could be an angle $\varphi=\arctan q_{1} / q_{2}$, because solutions of the compatibility equations (2.11) and (2.15) have the following form

$$
V\left(q_{1}, q_{2}\right)=F_{1}(\rho)+\frac{F_{2}(\varphi)}{\rho^{2}}, \quad \rho=r \quad \text { or } \quad \rho=\hat{r} .
$$

Cartesian coordinates. Separable in the Cartesian coordinates solution of the compatibility condition (2.11) reads as

$$
V\left(q_{1}, q_{2}\right)=F_{1}\left(q_{1}\right)+F_{2}\left(q_{2}\right) .
$$

In nonholonomic case characteristic tensor $\hat{\mathbf{K}}=K^{(1)} \hat{\mathrm{g}}^{-1}$ yields only one coordinate

$$
\hat{\varrho}=\sqrt{\frac{1+q_{1}^{2}}{1+q_{1}^{2}+q_{2}^{2}}}
$$

and the separable solution of the compatibility condition (2.15) looks like

$$
V\left(q_{1}, q_{2}\right)=F_{1}(\hat{\varrho})+\frac{F_{2}(\hat{\phi})}{\hat{\varrho}^{2}}, \quad \hat{\phi}=\arctan q_{1} .
$$

Thus, after nonholonomic reduction one gets separable potential, which is different, even in the form of standard potential (2.22) separable in Cartesian coordinates.

Summing up, we have found four characteristic coordinate systems for the Bertrand-Darboux type equation (2.18) associated with the integrable non-Hamiltonian vector field $\hat{X}(2.7)$. Additional first integral of this field $\hat{X}$ is a polynomial of the second order in momenta defined by the standard Killing tensor on the plane. Consequently, we can directly apply the standard Stäckel theory to the nonholonomic Heisenberg type systems. 


\section{Stäckel systems}

In Hamiltonian case there is a one-to-one correspondence between the so-called Stäckel systems, integrable Killing tensors which mutually commute in the algebraic sense and separation of variables [3, 14].

The nondegenerate $n \times n$ Stäckel matrix $S$, which $j$ column depends only on variable $u_{j}$

$$
\operatorname{det} S \neq 0, \quad \frac{\partial S_{k j}}{\partial u_{m}}=0, \quad j \neq m
$$

defines $n$ functionally independent integrals of motion

$$
H_{k}=\sum_{j=1}^{n} C_{j k}\left(p_{u_{j}}^{2}+U_{j}\left(u_{j}\right)\right), \quad C=S^{-1},
$$

which are in involution with respect to canonical Poisson brackets

$$
\left\{u_{i}, p_{u_{j}}\right\}=\delta_{i j}, \quad\left\{u_{i}, u_{j}\right\}=\left\{p_{u_{i}}, p_{u_{j}}\right\}=0 .
$$

The common level surface of the first integrals $H_{1}=\alpha_{1}, \ldots, H_{2}=\alpha_{n}$ is diffeomorphic to the $n$-dimensional real torus and one immediately gets

$$
p_{u_{j}}^{2}=\sum_{k=1}^{n} \alpha_{k} S_{k j}\left(u_{j}\right)-U_{j}\left(u_{j}\right) .
$$

It allows us to calculate quadratures for the corresponding Hamiltonian vector field $X=P d H_{1}$ :

$$
\sum_{j=1}^{n} \int_{\gamma_{0}\left(p_{0}, q_{0}\right)}^{\gamma_{j}\left(p_{u_{j}}, u_{j}\right)} \frac{S_{k j}(u) d u}{\sqrt{\sum_{k=1}^{n} \alpha_{k} S_{k j}(u)-U_{j}(u)}}=\beta_{k}, \quad k=1, \ldots, n,
$$

where $\beta_{1}=t$ and $\beta_{2}, \ldots, \beta_{n}$ are constants of integration. Solution of the problem is thus reduced to solving a sequence of one-dimensional problems, which is the essence of the method of separation of variables [3].

\subsection{Stäckel matrices for reduced systems}

In the definition of the Stäckel integrals of motion (3.1) momenta $p_{u}$ have to be canonically conjugated to eigenvalues $u$ of the characteristic $(1,1)$ tensor $\mathbf{K}$. For instance, if we take tensor $K^{(3)}(2.19)$ the eigenvalues of $\mathbf{K}$ are parabolic coordinates $\zeta_{1,2}(2.20)$ and the corresponding momenta read as

$$
p_{\zeta_{1,2}}=\frac{p_{1}}{2} \pm \frac{\sqrt{q_{1}^{2}+q_{2}^{2}} \mp q_{1}}{2 q_{2}} p_{2}
$$

In nonholonomic case the eigenvalues of $\hat{\mathbf{K}}$ are given by $\hat{\zeta}_{1,2}(2.21)$ and the conjugated momenta have the following form

$$
p_{\hat{\zeta}_{1,2}}=\frac{q_{2}^{2}+1 \pm q_{1} \sqrt{1+q_{2}^{2}} \sqrt{q_{1}^{2}+q_{2}^{2}}}{2\left(1-q_{1}^{2}\right)} p_{1}-\frac{q_{1} \mp \sqrt{1+q_{2}^{2}} \sqrt{q_{1}^{2}+q_{2}^{2}}}{2 q_{2}} p_{2} .
$$

In similar manner we can calculate all the canonical variables associated with tensors $K^{(j)}(2.19)$ and to obtain standard Stäckel matrices in Hamiltonian case

$$
S^{(1)}=\left(\begin{array}{cc}
1 & 1 \\
1 & -1
\end{array}\right), \quad S^{(2)}=\left(\begin{array}{cc}
0 & 1 \\
1 & -r^{-2}
\end{array}\right), \quad S^{(3)}=\left(\begin{array}{cc}
1 & 1 \\
-\zeta_{1}^{-1} & -\zeta_{2}^{-1}
\end{array}\right)
$$


and

$$
S^{(4)}=\left(\begin{array}{cc}
\frac{1}{\xi_{1}-\kappa^{2}} & \frac{1}{\xi_{2}-\kappa^{2}} \\
\frac{-1}{\xi_{1}\left(\xi_{1}-\kappa^{2}\right)} & \frac{-1}{\xi_{2}\left(\xi_{2}-\kappa^{2}\right)}
\end{array}\right) \text {. }
$$

For the nonholonomic Heisenberg type systems associated with tensors $K^{(j)}(2.19)$ Stäckel matrices are equal to

$$
\hat{S}^{(1)}=\left(\begin{array}{cc}
0 & \frac{1}{\hat{\varrho}^{2}\left(1-\hat{\varrho}^{2}\right)} \\
1 & \frac{-1}{\hat{\varrho}^{4}\left(1-\hat{\varrho}^{2}\right)}
\end{array}\right), \quad \hat{S}^{(2)}=\left(\begin{array}{cc}
0 & \frac{1}{1-\hat{r}^{2}} \\
1 & \frac{-1}{\hat{r}^{2}\left(1-\hat{r}^{2}\right)}
\end{array}\right)
$$

and

$$
\begin{aligned}
\hat{S}^{(3)} & =\left(\begin{array}{cc}
\frac{1}{1-\hat{\zeta}_{1}} & \frac{1}{1-\hat{\zeta}_{2}} \\
\frac{-1}{\hat{\zeta}_{1}\left(1-\hat{\zeta}_{1}\right)} & \frac{-1}{\hat{\zeta}_{2}\left(1-\hat{\zeta}_{2}\right)}
\end{array}\right), \\
\hat{S}^{(4)} & =\left(\begin{array}{cc}
\frac{1}{\left(\hat{\xi}_{1}-\kappa^{2}\right)\left(1-\hat{\xi}_{1}\right)} & \frac{1}{\left(\hat{\xi}_{2}-\kappa^{2}\right)\left(1-\hat{\xi}_{2}\right)} \\
\frac{-1}{\hat{\xi}_{1}\left(\hat{\xi}_{1}-\kappa^{2}\right)\left(1-\hat{\xi}_{1}\right)} & \frac{-1}{\hat{\xi}_{2}\left(\hat{\xi}_{2}-\kappa^{2}\right)\left(1-\hat{\xi}_{2}\right)}
\end{array}\right) .
\end{aligned}
$$

It is easy to see, that imposing linear, non integrable constraint (1.7) in polar, parabolic and elliptic cases we have to multiply $j$ column of the standard Stäckel matrices on the function $\left(1-u_{j}\right)$, where $u_{j}$ is the eigenvalue of $\hat{\mathbf{K}}$. Similar transformations of the Stäckel matrices for nonholomic systems on a two-dimensional sphere are discussed in [22].

Using a new time variable $\tau$ defined by

$$
d t=\mu^{-1} d \tau \equiv\left(1+q_{1}^{2}+q_{2}^{2}\right) d \tau
$$

we can rewrite the conformally Hamiltonian vector field $\hat{X}(2.7)$ in the Hamiltonian form and obtain quadratures similar to (3.2).

Thus, in nonholonomic case solution of the problem is reduced to solving a sequence of one-dimensional problems after suitable change of time.

\subsection{Compatible Poisson brackets}

Riemannian geometry is not, a priori, concerned with symplectic or Poisson structures. Nevertheless, it is known that any tensor field $L$ with vanishing Nijenhuis torsion on the Riemannian manifold $Q$ yields trivial deformation of the canonical Poisson bracket $\{\cdot, \cdot\}$ on its cotangent bundle $T^{*} Q$

$$
\left\{q_{i}, q_{j}\right\}_{L}=0, \quad\left\{q_{i}, p_{j}\right\}_{L}=-L^{i j}, \quad\left\{p_{i}, p_{j}\right\}_{L}=\left(\frac{\partial L^{k j}}{\partial q_{i}}-\frac{\partial L^{k i}}{\partial q_{j}}\right) p_{k}
$$

where $\left(p_{i}, q_{i}\right)$ are fibered coordinates. This Poisson bracket is compatible with the canonical one, so that there is a recursion operator $N=P_{L} P^{-1}$, which allows us to construct a whole family of compatible brackets associated with Poisson bivectors $P_{L}^{(m)}=N^{m} P$ on $T^{*} Q$ [21, 22]. 
According to [3] generic solution of the Killing equation $K(2.16)$ determines a conformal Killing tensor with vanishing Nijenhuis torsion

$$
L=K-\operatorname{tr}(K) g
$$

and Turiel's deformation (3.3) of the canonical Poisson bracket [21, 22]. The eigenvalues of the corresponding recursion operator $N=P_{L} P^{-1}$ are characteristic coordinates. Integrals of motion $H_{1}(2.8)$ and $H_{2}(2.9)$ are in involution with respect to the compatible Poisson brackets

$$
\left\{H_{1}, H_{2}\right\}=\left\{H_{1}, H_{2}\right\}_{L}=0
$$

and to other polynomial Poisson brackets associated with the Poisson bivectors $P_{L}^{(m)}$. Indeed, we can find such $L$-tensors directly from the Hamilton function $H_{1}$ using modern software [16].

For the Heisenberg type systems, we have to solve the same Killing equation (2.14) and second equation on potential with $(1,1)$ tensor $\hat{\mathbf{K}}=K \hat{\mathrm{g}}$. This tensor does not appear in the Turiel construction of the Poisson bracket and we can directly prove the following proposition.

Proposition 3. Integrals of motion $\hat{H}_{1}(2.12)$ and $\hat{H}_{2}$ (2.13) are in involution

$$
\left\{\hat{H}_{1}, \hat{H}_{2}\right\}^{\wedge}=\left\{\hat{H}_{1}, \hat{H}_{2}\right\}_{L}=0
$$

with respect to the compatible Poisson brackets $\{\cdot, \cdot\}^{\wedge}(2.5)$ and $\{\cdot, \cdot\}_{L}(3.3)$. The eigenvalues of the recursion operator

$$
\hat{N}=P_{L} \hat{P}^{-1},
$$

where $\hat{P}$ is given by (2.7), are characteristic coordinates discussed in the previous Section.

The proof consists of the straightforward calculations.

Thus, starting with a common Poisson bivector $P_{L}$ we can construct two recursion operators $N$ and $\hat{N}$ and two families of the Poisson brackets on $T^{*} \mathbb{R}^{2}$.

\subsection{Lax matrices}

We can construct Lax representations for the Stäckel systems with uniform rational and polynomial potentials $U_{j}=U$ in (3.1), see [13] and references within. For the sake of brevity we consider only systems associated with tensor $K^{(3)}(2.19)$.

If $V\left(q_{1}, q_{2}\right)=0$, we can introduce a well-known $2 \times 2$ Lax matrix for the geodesic motion on the plane

$$
\mathcal{L}(u)=\left(\begin{array}{cc}
h & e \\
f & -h
\end{array}\right)(u) \equiv\left(\begin{array}{cc}
\frac{1}{2} \frac{d e(u)}{d t} & e(u) \\
-\frac{1}{2} \frac{d^{2} e(u)}{d t^{2}} & -\frac{1}{2} \frac{d e(u)}{d t}
\end{array}\right),
$$

which satisfies to the Lax equation

$$
\frac{d}{d t} \mathcal{L}=[\mathcal{L}, \mathcal{A}] \equiv \mathcal{L} \mathcal{A}-\mathcal{A L}, \quad \text { with } \quad \mathcal{A}=\left(\begin{array}{ll}
0 & 1 \\
0 & 0
\end{array}\right)
$$

This equation guarantees that the eigenvalues of $\mathcal{L}$ are conserved quantities in the involution.

In our case function $e(u)$ depends on the so-called spectral parameter $u$

$$
e(u)=\frac{\left(u-\zeta_{1}\right)\left(u-\zeta_{2}\right)}{u}=u-2 q_{1}-\frac{q_{2}^{2}}{u},
$$


which is completely defined by the eigenvalues of the characteristic tensor $\mathbf{K}=K^{(3)} \mathrm{g}^{-1}$ or parabolic coordinates $\zeta_{1,2}$ on the plane.

The involution property of eigenvalues of the Lax matrix $\mathcal{L}$ is equivalent to existence of a classical $r$-matrix $r_{12}$, that as

$$
\left\{\mathcal{L}_{1}(u), \mathcal{L}_{2}(v)\right\}=\left[r_{12}(u, v), \mathcal{L}_{1}(u)\right]-\left[r_{21}(u, v), \mathcal{L}_{2}(v)\right] .
$$

Here we use the familiar notation for tensor product of $\mathcal{L}$ and unit matrix $I$

$$
\mathcal{L}_{1}(u)=\mathcal{L}(u) \otimes \mathrm{I}, \quad \mathcal{L}_{2}(v)=\mathrm{I} \otimes \mathcal{L}(v), \quad r_{21}(u, v)=\Pi r_{12}(v, u) \Pi,
$$

whereas $\Pi$ is the permutation operator: $\Pi x \otimes y=y \otimes x, \forall x, y$. Evaluating canonical Poisson brackets (2.3) between entries of the Lax matrix $\mathcal{L}(u)(3.4)$ we obtain a constant $r$-matrix

$$
r_{12}(u, v)=\frac{2}{v-u} \Pi \equiv \frac{2}{v-u}\left(\begin{array}{cccc}
1 & 0 & 0 & 0 \\
0 & 0 & 1 & 0 \\
0 & 1 & 0 & 0 \\
0 & 0 & 0 & 1
\end{array}\right) .
$$

If we want to consider separable in parabolic coordinates potentials $V\left(q_{1}, q_{2}\right)$, we have to add some items to the initial Lax matrix $\mathcal{L}(u)(3.4)$. For instance, we can take the following additive perturbation

$$
\mathcal{L}_{V}(u)=\mathcal{L}(u)+\left(\begin{array}{cc}
0 & 0 \\
\Delta f & 0
\end{array}\right), \quad \Delta f=\left[\phi(u) e^{-1}(u)\right]
$$

where $\phi(u)$ is an arbitrary constant function and $[g(u)]$ is a truncated Laurent series expansion of $g(u)$ with respect to the variable $u$. For instance, when $\phi(u)=-a u^{3}$ the Taylor part of expansion about $u=\infty$ looks like

$$
\left[-a u^{3} e^{-1}(u)\right]=-a\left(u^{2}+2 u q_{1}+q_{2}^{2}+4 q_{1}^{2}\right) .
$$

Substituting this expression into $\mathcal{L}_{V}(u)$ one gets a Lax matrix for the Hénon-Heiles system on the plane with the Hamiltonian

$$
H_{1}=\frac{1}{2}\left(p_{1}^{2}+p_{2}^{2}\right)+8 a q_{1}\left(2 q_{1}^{2}+q_{2}^{2}\right) .
$$

In nonholonomic case we can also find similar Lax matrices, classical $r$-matrix and integrable potentials.

Proposition 4. For the Heisenberg type systems equations of motion $(2.7)$ when $V\left(q_{1}, q_{2}\right)=0$ can be rewritten in the Lax form (3.5) if

$$
\hat{\mathcal{L}}(u)=\left(\begin{array}{cc}
\hat{h} & \hat{e} \\
\hat{f} & -\hat{h}
\end{array}\right)(u) \equiv\left(\begin{array}{cc}
\frac{1}{2 \mu} \frac{d \hat{e}(u)}{d t} & \hat{e}(u) \\
-\frac{d}{\mu d t}\left(\frac{1}{2 \mu} \frac{d \hat{e}(u)}{d t}\right)-2 \hat{H}_{1} \hat{e}(u) & -\frac{1}{2 \mu} \frac{d \hat{e}(u)}{d t}
\end{array}\right)
$$

and

$$
\hat{\mathcal{A}}=\left(\begin{array}{cc}
0 & \mu \\
-2 \mu \hat{H}_{1} & 0
\end{array}\right) .
$$

Here $\mu=\left(1+q_{1}^{2}+q_{2}^{2}\right)^{-1}$ is a conformal factor (2.7) and function

$$
\hat{e}(u)=\frac{\left(u-\hat{\zeta}_{1}\right)\left(u-\hat{\zeta}_{2}\right)}{u}=u-\frac{2 q_{1}}{1+q_{1}^{2}+q_{2}^{2}}-\frac{q_{2}^{2}}{u\left(1+q_{1}^{2}+q_{2}^{2}\right)}
$$

is completely defined by characteristic coordinates $\hat{\zeta}_{1,2}(2.21)$. 
The Lax matrix (3.7) was constructed by using the generic construction of the Lax matrices for the Stäckel systems [13]. The modification consists only of application of the nontrivial conformal factor $\mu$.

Evaluating Poisson brackets $\{\cdot, \cdot\}^{\wedge}(2.5)$ between entries of the Lax matrix $\hat{\mathcal{L}}(u)(3.7)$ we find the corresponding classical $r$-matrix

$$
\hat{r}_{12}(u, v)=r_{12}(u, v)+2\left(\begin{array}{cccc}
0 & 0 & 0 & 0 \\
0 & 0 & \hat{e}(v) & 0 \\
0 & 0 & 0 & 0 \\
-\hat{f}(v) & 0 & 0 & 0
\end{array}\right),
$$

which explicitly depends on dynamical variables via entries of $\hat{\mathcal{L}}(u)(3.7)$.

As above, we can consider additive perturbation of this Lax matrix

$$
\hat{\mathcal{L}}_{V}(u)=\hat{\mathcal{L}}(u)+\left(\begin{array}{cc}
0 & 0 \\
\Delta \hat{f} & 0
\end{array}\right), \quad \Delta \hat{f}=\left(u^{2}-1\right)\left[\phi(u) \hat{e}^{-1}(u)\right]
$$

in order to get Lax matrices for the nonholonomic Heisenberg type systems with $V \neq 0$. For instance, when $\phi(u)=-a u^{3}$ the Teylor part of expansion about $u=\infty$ looks like

$$
\left[-a u^{3} \hat{e}^{-1}(u)\right]=-a\left(u^{2}+\frac{2 u q_{1}}{1+q_{1}^{2}+q_{2}^{2}}+\frac{q_{2}^{2}}{1+q_{1}^{2}+q_{2}^{2}}+\frac{4 q_{1}^{2}}{\left(1+q_{1}^{2}+q_{2}^{2}\right)^{2}}\right) .
$$

Substituting this expression into $\hat{\mathcal{L}}_{V}(u)$ one gets a Lax matrix for the nonholonomic counterpart of the Hénon-Hieles system on the plane with the Jacobi integral

$$
\hat{H}_{1}=\frac{1}{2}\left(p_{1}^{2}+p_{2}^{2}+\left(q_{2} p_{1}-q_{1} p_{2}\right)^{2}\right)+\frac{8 a q_{1}\left(q_{1}^{2} q_{2}^{2}+q_{2}^{4}+2 q_{1}^{2}+q_{2}^{2}\right)}{\left(1+q_{1}^{2}+q_{2}^{2}\right)^{3}} .
$$

\section{Conclusion}

Imposing nonholonomic constraints to Hamiltonian systems and making a suitable reduction one gets some special class of non Hamiltonian systems on the reduced phase space, see book [7]. Usually we can not investigate the reduced system using standard mathematical methods of Hamiltonian dynamics. For instance, we do not know how to get Lax matrices, classical $r$ matrices or compatible Poisson brackets for the conformally Hamiltonian systems associated with the nonholonomic Chaplygin ball, nonholonomic Suslov or Veselova systems.

In this note we find Killing tensors and compatible Poisson brackets, describe integrable potentials and characteristic coordinates, evaluate Stäckel matrices and Stäckel quadratures, to show Lax matrices and classical $r$-matrices for the nonholonomic Heisenberg type systems. Indeed, we prove that some modern geometric methods of Hamiltonian mechanics can be directly applied to the reduced conformally Hamiltonian systems. Other similar examples can be found in $[6,8,20,22,23]$.

Of course, we can impose other nonholonomic constraints on the original Hamiltonian system (1.1), (1.2). It may be interesting to describe all the constraints which lead to nontrivial integrable metrics and potentials on the reduced phase space.

\section{Acknowledgements}

We are very grateful to the referees for thorough analysis of the manuscript, constructive suggestions and proposed corrections, which certainly lead to a more profound discussion of the results. We are also deeply grateful A.V. Borisov and I.A. Bizayev for the relevant discussion. 
Section 2 was written by A.V. Tsiganov and supported by the Russian Science Foundation (project 15-12-20035). Section 3 was written by Yu.A. Grigoryev and A.P. Sozonov within the framework of the Russian Science Foundation (project 15-11-30007).

\section{References}

[1] Ankiewicz A., Pask C., The complete Whittaker theorem for two-dimensional integrable systems and its application, J. Phys. A: Math. Gen. 16 (1983), 4203-4208.

[2] Bates L., Śniatycki J., Nonholonomic reduction, Rep. Math. Phys. 32 (1993), 99-115.

[3] Benenti S., Orthogonal separable dynamical systems, in Differential Geometry and its Applications (Opava, 1992), Math. Publ., Vol. 1, Editors O. Kowalsky, D. Krupka, Silesian University Opava, Opava, 1993, 163184.

[4] Bertrand J.M., Mémoire sur quelques-unes des forms les plus simples que puissent présenter les intégrales des équations différentielles du mouvement d'un point matériel, J. Math. Pures Appl. 2 (1857), 113-140.

[5] Bizyaev I.A., Borisov A.V., Mamaev I.S., Hamiltonization of elementary nonholonomic systems, Russ. J. Math. Phys. 22 (2015), 444-453, arXiv:1601.00884.

[6] Bizyaev I.A., Tsiganov A.V., On the Routh sphere problem, J. Phys. A: Math. Theor. 46 (2013), 085202, 11 pages, arXiv:1210.7903.

[7] Bloch A.M., Nonholonomic mechanics and control, Interdisciplinary Applied Mathematics, Vol. 24, SpringerVerlag, New York, 2003.

[8] Borisov A.V., Mamaev I.S., Symmetries and reduction in nonholonomic mechanics, Regul. Chaotic Dyn. 20 (2015), 553-604.

[9] Borisov A.V., Mamaev I.S., Bizyaev I.A., The hierarchy of dynamics of a rigid body rolling without slipping and spinning on a plane and a sphere, Regul. Chaotic Dyn. 18 (2013), 277-328.

[10] Borisov A.V., Mamaev I.S., Tsiganov A.V., Non-holonomic dynamics and Poisson geometry, Russ. Math. Surv. 69 (2014), 481-538.

[11] Cushman R., Duistermaat H., Śniatycki J., Geometry of nonholonomically constrained systems, Advanced Series in Nonlinear Dynamics, Vol. 26, World Sci. Publ. Co. Pte. Ltd., Hackensack, NJ, 2010.

[12] Darboux G., Sur un probléme de mécanique, Arch. Néerl. 6 (1901), 371-376.

[13] Eilbeck J.C., Enol'skii V.Z., Kuznetsov V.B., Tsiganov A.V., Linear $r$-matrix algebra for classical separable systems, J. Phys. A: Math. Gen. 27 (1994), 567-578, hep-th/9306155.

[14] Eisenhart L.P., Separable systems of Stäckel, Ann. of Math. 35 (1934), 284-305.

[15] Fassò F., Sansonetto N., Conservation of energy and momenta in nonholonomic systems with affine constraints, Regul. Chaotic Dyn. 20 (2015), 449-462, arXiv:1505.01172.

[16] Grigoryev Yu.A., Tsiganov A.V., Symbolic software for separation of variables in the Hamilton-Jacobi equation for the L-systems, Regul. Chaotic Dyn. 10 (2005), 413-422, nlin.SI/0505047.

[17] Kozlov V.V., The Euler-Jacobi-Lie integrability theorem, Regul. Chaotic Dyn. 18 (2013), 329-343.

[18] Molina-Becerra M., Galán-Vioque J., Freire E., Dynamics and bifurcations of a nonholonomic Heisenberg system, Internat. J. Bifur. Chaos Appl. Sci. Engrg. 22 (2012), 1250040, 14 pages.

[19] Smirnov R.G., On the classical Bertrand-Darboux problem, J. Math. Sci. 151 (2008), 3230-3244, math-ph/0604038.

[20] Tsiganov A., Integrable Euler top and nonholonomic Chaplygin ball, J. Geom. Mech. 3 (2011), 337-362, arXiv:1002.1123.

[21] Tsiganov A.V., On bi-integrable natural Hamiltonian systems on Riemannian manifolds, J. Nonlinear Math. Phys. 18 (2011), 245-268, arXiv:1006.3914.

[22] Tsiganov A.V., One family of conformally Hamiltonian systems, Theoret. Math. Phys. 173 (2012), 14811497, arXiv:1206.5061.

[23] Tsiganov A.V., On integrable perturbations of some nonholonomic systems, SIGMA 11 (2015), 085, 19 pages, arXiv:1505.01588.

[24] van der Schaft A.J., Maschke B.M., On the Hamiltonian formulation of nonholonomic mechanical systems, Rep. Math. Phys. 34 (1994), 225-233.

[25] Whittaker E.T., A treatise on the analytical dynamics of particles and rigid bodies, Cambridge Mathematical Library, Cambridge University Press, Cambridge, 1988. 